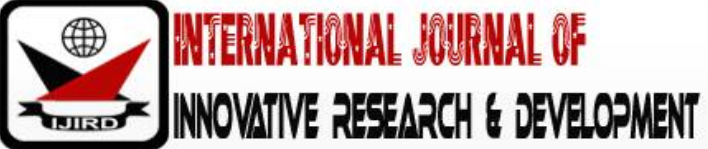

ISSN 2278 - 0211 (Online)

\section{Computational Fluid Dynamics (CFD) Transient Pressure and Temperature Simulation of a Natural Gas- Hydrogen Gas Blend Transportation Pipeline}

\author{
Oghenethoja Monday Umuteme \\ CEO, Derock Global Energy Resources Company Limited, Port Harcourt, Nigeria
}

\begin{abstract}
:
The need for a CFD exploratory investigation into the possible effect of hydrogen injection into existing natural gas pipeline network, was the driver for this study. Recent technical feasibility reports on transporting a blend of both gases (JIR, 2019), did not mention if a study was done to rule out the possible effect of natural gas-hydrogen blend on hydrate formation in a hydrate-prone gas pipeline. A CFD model of $0.0508 m$ and $10 \mathrm{~m}$ length, with a mesh size of 20,000 elements was developed and simulated using ANSYS Fluent version R19.3. Hydrogen concentration by volume of 5\%, $10 \%, 15 \%$ and $20 \%$ were simulated at velocities of $5 \mathrm{~m} / \mathrm{s}$ and $10 \mathrm{~m} / \mathrm{s}$. The results indicated that increasing the volume concentration of hydrogen gas in the blend led to a reduction of downstream temperature. Again, the force on the inner wall decreased as the volume of hydrogen injected increased. The inference was that the reduction in temperature could lead to hydrates formation, whereas the reduction in exerted force on the pipe wall by the fluid could suggest a reduction in turbulence. However, this study was exploratory in nature and it is recommended that a detailed experimental study be carried out to clearly define the hydraulic and thermal behavior of a pipeline transporting a natural gas-hydrogen blend under various subcooling temperatures.
\end{abstract}

Keywords: Natural gas, hydrogen gas, natural gas-hydrogen blend, transportability, pressure, temperature

\section{Introduction}

The world is moving towards greener sources of energy, and hydrogen gas as fuel for automobile and electricity generation is one option that has attracted much research. From production to utilisation of hydrogen fuel in automobiles and electricity generating plants the challenge has been storage and transportability. Whereas hydrogen can be extracted from methane through steam methane reforming (SMR) process (Melaina, Antonia, \&Penev, 2013), yet the hydrogen retrieved from a singular pipeline is small compared with industrial demand. Extracting hydrogen is not economical on a small scale. Hence, the need to have an industrial plant is the best option, from where it is transported to end-users. However, there is current interest in injecting hydrogen gas into existing natural gas pipeline networks to form a 'natural gas-hydrogen' (NGHY) blend in order to minimise the initial cost associated with the installation of pipelines (Meng et al, 2017). Possible success below $20 \%$ by volume of hydrogen gas injected into natural gas pipeline has been reported to have non-significant associated risk (Melaina, Antonia, \& Penev, 2013). A joint industry report by operators recommended a short-term volume of $6 \%$ injection into existing natural gas network in order to avoid any significant risk, while studies to ascertain the most economical and technically feasible injection volume in the long term is been researched (JIR, 2019).

Some studies have already reported the hazard associated with pipe rupture (e.g., Meng et al, 2017) releasing hydrogen gas into the environment. Again, Kaczynski et al. (2019) has reported the effect of increasing hydrogen fraction blend in natural gas pipelines on transportability suggesting that volume fraction of hydrogen beyond $10 \%$ can negatively affect pressure drop. But, the concern for this study is the possibility of methane hydrates forming in the pipeline if the temperature drops below the methane hydrate loci at high pressures. Hence, this work seeks to use CFD to assess the effect of increasing hydrogen gas volume on the transient temperature and pressure response of the pipeline. It is anticipated that the outcome of this study will encourage experimental study of the possibility of hydrates formation in NGHY blend and the hydrogen volume fraction when this can occur.

\section{Literature Review}

CFD software has been used to model transport behavior of fluids in pipelines in order to predict temperature and pressure behavior, and other physical phenomenon such as vibration and hydrates. Evidence in the literature include CFD studies on hydrates formation in gas dominated pipeline system (Neto et al, 2016); on the transportability of gas-solid and liquid mixture (e.g., Sultan et a; 2017); on flow behavior in bends - including vortex induced vibrations (e.g., Jozian\&Vafajoo, 2018; Jujuly, 2017), etc. CFD studies on hydrogen transporting pipelines are currently on risk of failure (Meng et al, 2017). The hydraulic simulation studies by Kaczynski et al. (2019) and Wlodek et al. (2016) indicated that up 
to $10 \%$ by molar volume fraction of hydrogen in natural gas blend can be transported based on pressure drop estimations. To the knowledge of this author from literature searches and study, there is no study that explain the temperature and pressure response due to increasing hydrogen gas volume in a natural gas-hydrogen blend transported through pipeline, and the possibility of hydrates formation due to temperature drop. Consequently, this study is an exploratory CFD study to model the effect of temperature and pressure response of the gas pipeline as the volume of hydrogen injected to form the natural gas-hydrogen blend increases. Early explanation of two-phase flow models and methods can be found in the literature (Thorley \&Tiley, 1987; Stewart \&Wendroff, 1984). The increasing use of these models can be attested in the recent volumes of literature on multiphase CFD and analytical studies.

\section{Methodology}

This exploratory CFD model was developed and simulated using ANSYS Fluent R19.3. Though, multiphase flow in CFD can be modelled using the eulerian or langragian models, this CFD is modelled using the eulerian 'Mixture' governing equations with no mass transfer between the phases. This is because, the blend is treated as a homogenous fluid.

\subsection{Computational Domain}

The computational domain is a 2-D horizontal section of a pipeline of $0.0508 \mathrm{~m}$ diameter and $10 \mathrm{~m}$ length. Mesh size was 20,000 cells with inflation at the wall to capture near-wall turbulent effects.

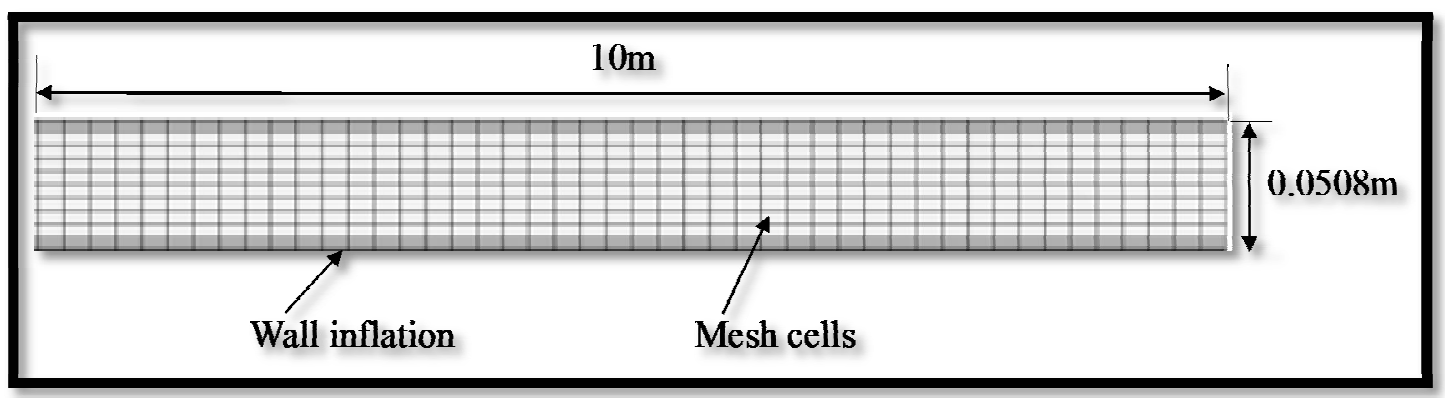

Figure 1: Sectional Representation of the Meshed Computational Domain

\subsection{Governing Equations}

Though hydrogen is lighter and expected to travel faster than natural gas, a local equilibrium has been assumed for the blend, hence slip velocity between both phases is assumed to 'zero.' The 'mixture' multiphase model can be used to model homogeneous multiphase flows where there is very strong coupling and the phases are moving at the same velocity and for estimating non-Newtonian viscosity ANSYS theory guide. The following sets of 1-D transport governing equations are employed based on the mixture model and specific assumptions for this study:

Mass Continuity: There is no mass transfer between the phases, hence the mass continuity equation can be stated as:

$\frac{\partial}{\partial t}\left(\rho_{m}\right)+\nabla \cdot\left(\rho_{m} \vec{v}_{m}\right)=0$

Where: $\vec{v}_{m}$ is mass-averaged velocity vector, defined as $\vec{v}_{m}=\frac{\alpha_{N_{-} G} \rho_{N_{-} G} \vec{v}_{N_{-} G}+\alpha_{H_{-} G} \rho_{H_{-} G} \vec{v}_{H_{-} G}}{\rho_{m}} ; \rho_{m}$ is the mixture density $\left(\mathrm{kg} / \mathrm{m}^{3}\right)$, defined as $\alpha_{N_{-} G} \rho_{N_{-} G}+\alpha_{H_{-} G} \rho_{H_{-} G} ; \alpha_{N_{-} G}$ is the phase volume fraction of natural gas; $\alpha_{H_{-} G}$ is the phase volume fraction of hydrogen gas; $\rho_{N_{-} G}$ is the density of natural gas $\left(\mathrm{kg} / \mathrm{m}^{3}\right)$; and $\rho_{H_{-} G}$ is the density of hydrogen gas $\left(\mathrm{kg} / \mathrm{m}^{3}\right)$.

Momentum: It is assumed that there is no body force and no gravity effect, no drift velocity for the secondary phase. The momentum equation can be written as:

$\frac{\partial}{\partial t}\left(\rho_{m} \vec{v}_{m}\right)+\nabla \cdot\left(\rho_{m} \vec{v}_{m} \vec{v}_{m}\right)=-\nabla p+\nabla \cdot\left[\mu_{m}\left(\nabla \vec{v}_{m}+\nabla \vec{v}_{m}^{T}\right)\right]$

Where: $p$ is system pressure (Pa.); $\mu_{m}$ is mixture viscosity (Pa.s); and the expression $\mu_{m}\left(\nabla \vec{v}_{m}+\nabla \vec{v}^{T}{ }_{m}\right)$ is the shear stress term (Pa.m). Other variables are as defined earlier.

Energy: There are no external volumetric heat sources, hence the energy equation is reduced to the form:

$\frac{\partial}{\partial t} \sum_{k=1}^{n}\left(\alpha_{k} \rho_{k} E_{k}\right)+\nabla \cdot \sum_{k=1}^{n}\left(\alpha_{k} \vec{v}_{k}\left(\rho_{k} E_{k}+p\right)\right)=\nabla \cdot\left(k_{e f f} \nabla T\right)$

Where: $\alpha_{k}, \rho_{k}, E_{k} \& \vec{v}_{k}$ are the phase volume fraction (dimensionless), density $\left(\mathrm{kg} / \mathrm{m}^{3}\right.$ ), total energy (J) and velocity vector $(\mathrm{m} / \mathrm{s})$ respectively; $T$, is the temperature of the fluid mixture $(\mathrm{K}) ; k_{e f f}$ is the effective conductivity of the system $(\mathrm{W} / \mathrm{m}-\mathrm{K})$. 
This work is a CFD exploratory investigation into the possible effect on pressure and temperature of the system when hydrogen gas is injected into existing natural gas pipeline networks. Hence, the properties for natural gas and hydrogen gas used for this simulation are those defined for methane and hydrogen gas respectively, in ANSYS fluent version R19.3, without modifications. Input pressure for all simulations was 10MPa. Fluid blend temperature was set to $292 \mathrm{~K}$, while the pipe wall temperature was set to $285 \mathrm{~K}$, this was to create a subcooling temperature driving force for the blend. Simulations were carried out at $5 \mathrm{~m} / \mathrm{s}$ and $10 \mathrm{~m} / \mathrm{s}$ input velocities. Ambient pressure was $0.1 \mathrm{MPa}$.

\subsection{Model Setup}

Fluent solver configuration is pressure based, 2D planar, transient and absolute velocity formulation. The eulerian multiphase model is 'Mixture.' Volume fraction formulation is implicit, with secondary phase dispersed in the primary phase. The primary continuous phase is natural gas with $99 \%$ methane. The secondary hydrogen phase is dispersed and mixed in the primary phase at various volumetric concentration of 5\%,10\%, 15\% and 20\%. Drag force between the phases is assumed as 'zero.' Energy solver was engaged to account for heat transfer based on thermal gradient between the pipe wall and the NGHY blend. The turbulence energy and dissipation to take into effect the near wall turbulence effect was achieved using the kinetic-epsilon $(\mathrm{k}-\varepsilon)$ model, with realizable and enhanced wall treatment from pressure gradient and thermal effects, which was solved per phase.

The pressure-velocity coupling is 'SIMPLE,' and gradient set to 'Least Square Cell Based.' Pressure is 'PRESTO.' Pressure, momentum, turbulence kinetic energy and transient formulation were solved using second order upwind. Other parameters and solutions control 'under-relaxation factors' were left as default. Convergence criteria was set to 'Time Step convergence' with all conditions met, and residuals left as default. Initialization was achieved from inlet and simulation 'Run' was achieved at a time-step size of $0.1 \mathrm{~s}$ for 300 time-steps. For each model run at velocities of $5 \mathrm{~m} / \mathrm{s}$ and $10 \mathrm{~m} / \mathrm{s}$, the temperature contour, temperature gradient, and transient pressure and force exerted on the inner wall were recorded.

\section{Results}

\subsection{Effect of Hydrogen Gas Volume on Pipeline Temperature}

Temperature plays a significant role in the transport of gas in pipelines. This is because the formation of gas hydrates is dependent on the thermal driving force, which is accounted for by the subcooling temperature (Wang et al., 2018; Zhang, Wu, \& Mu, 2017; Sloan, Koh \& Sum, 2010). This effect was modelled in this study by assuming that the pipeline temperature is lower than that of the NGHY blend and the entire pipeline-fluid system is thermally disconnected from the ambient environment.

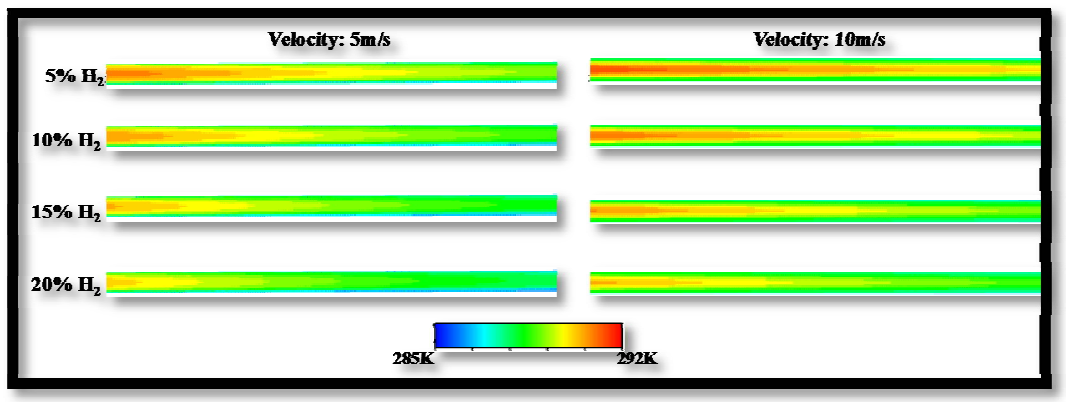

Figure 2: Temperature Contours Showing Effect of Hydrogen Gas Volume on Pipeline Temperature

The above temperature contours can be represented using the temperature gradient presented in Figure 3

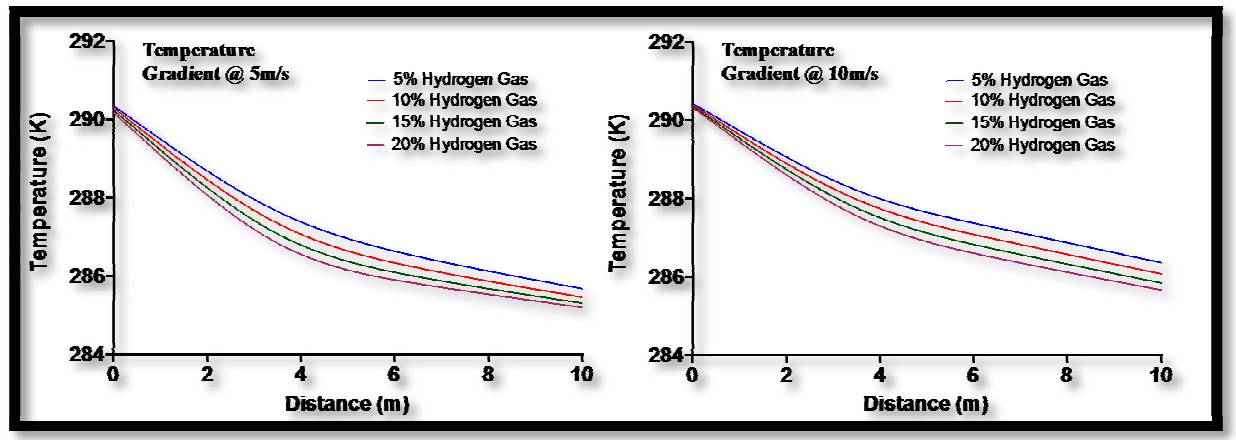

Figure 3: The Effect of Hydrogen Gas Volume on Pipeline Temperature at Mixture Velocity of $5 \mathrm{~m} / \mathrm{s}$ and $10 \mathrm{~m} / \mathrm{s}$.

The indication from figures 4.1 and 4.2 is that increasing the volume of hydrogen in the NG HY blend reduces the downstream temperature in the pipeline. Thus, hydrogen has a cooling effect on the natural gas in the pipeline. However, 
the observation in this study is inconsistent with the report in Kaczynski et al. (2019), where the temperature increases with increasing molar volume fraction of hydrogen in the blend. This may have occurred, because in their study the ambient temperature was in contact with the pipeline and higher by $10^{\circ} \mathrm{C}$ than the temperature of the NGHY blend in the pipeline. And, this could have led to the hydrogen gas, being lighter in mass, to increase in temperature faster, with a higher velocity than the natural gas. Whereas, in this CFD study the assumption is that the blend is a mixture with no slip in velocity between both gases at the inlet. Thus, allowing for a real blend between both gases as determined by the software.

\subsection{Effect of Hydrogen Gas Volume on Pipeline Pressure}

Pressure variation across a gas pipeline is also a determining factor on gas pipeline flow assurance. When the pressure drops below a certain threshold, there is the possibility of water drop-out on the inner pipe wall. The pressure response for this CFD study is presented below.

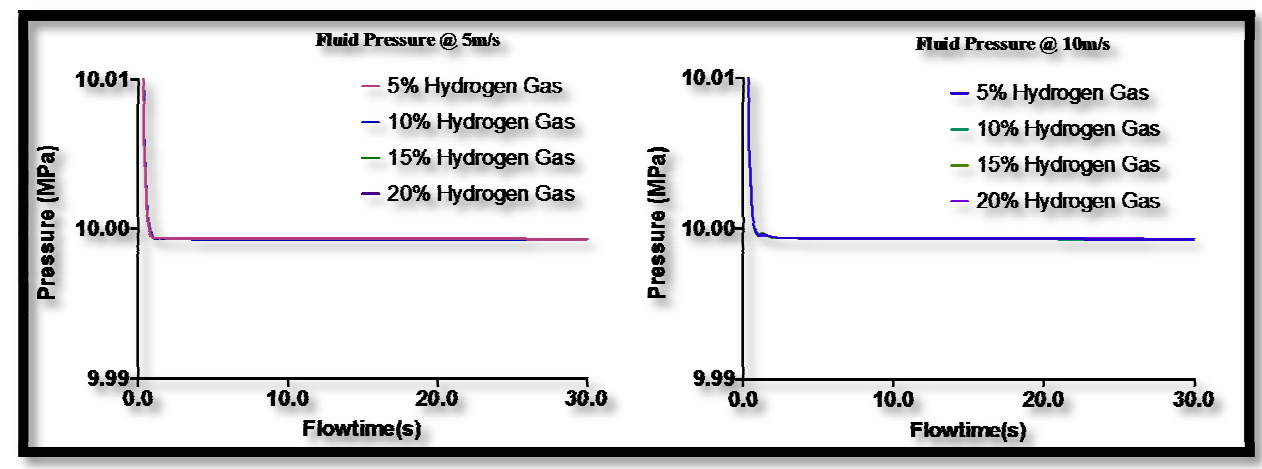

Figure 4: The Effect of Hydrogen Gas Volume on Pipeline Pressure

The relatively stable pressure response in Figure 4 is in agreement with literature evidence in Wlodek et al. (2016), that hydrogen volume up to $20 \%$ resulted in no significant pressure drop up to a pipeline distance of $50 \mathrm{~km}$ between fractions of hydrogen injected. This observation is also corroborated in a recent study by Kaczynski et al. (2019).

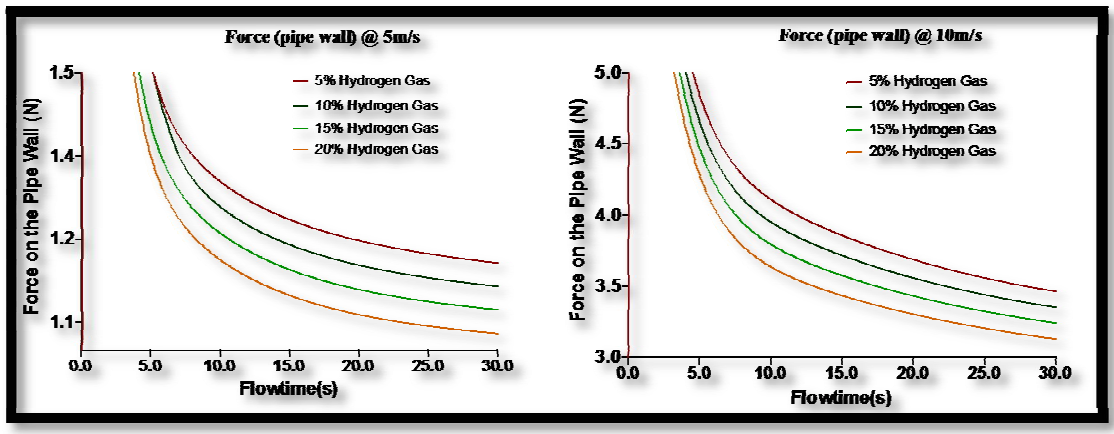

Figure 5: The Effect of Hydrogen Gas Volume on Fluid Force Exerted on the Pipe Wall

The indication in Figure 5 is in agreement with the study by Wlodek et al. (2016), that the friction coefficient increases with increasing hydrogen volume in the blend. Increasing friction coefficient increases pressure drop through loss of hydraulic energy, thus reducing the average force on the wall. This phenomenon is attributed to the reduction in density of the blend as hydrogen gas volume fraction increases, because hydrogen is lighter than natural gas (Kaczynski et al., 2019).

\section{Conclusion}

This study is an exploratory investigation into the possible effect of hydrogen injection into natural gas on temperature and pressure of the pipeline system using a CFD numerical approach. Recent technical feasibility report on transporting a blend of both gases did not mention if a study was done to rule out the possible effect of blending both gases on hydrate formation in a hydrate-prone gas pipeline. A CFD model of $0.0508 \mathrm{~m}$ and $10 \mathrm{~m}$ length, with a mesh size of 20,000 elements was developed in ANSYS Fluent version R19.3. Simulation was carried out with hydrogen concentration by volume of $5 \%, 10 \%, 15 \%$ and $20 \%$ at velocities of $5 \mathrm{~m} / \mathrm{s}$ and $10 \mathrm{~m} / \mathrm{s}$. The results indicated that increase in the volume concentration of hydrogen gas in the blend led to a reduction of downstream temperature in both velocity scenarios. Also, the force exerted on the inner wall from the blend decreased as the volume of hydrogen injected increases. The implication was that the reduction is temperature could lead to hydrates formation, whereas the reduction in exerted force on the pipe wall by the fluid could suggest a reduction in turbulence. However, because this study was exploratory in nature, it is recommended that a further experimental study be carried out to clearly define the hydraulic and thermal behavior of a pipeline transporting a natural gas-hydrogen blend. The author intends to carry out an accurate predictive study of the effect of hydrogen gas injection into existing hydrates-prone natural gas pipeline on hydrates deposition in future studies. 


\section{Acknowledgement and Declaration}

This study was made possible by Derock Global Energy Resources Company Limited, Port Harcourt, Nigeria. The author declared no conflict of interest.

\section{References}

i. JIR. (2019). Technical and economic conditions for injecting hydrogen into natural gas networks. Final report retrieved from https://www.grtgaz.com/fileadmin/plaquettes/en/2019/Technical-economic-conditions-forinjecting-hydrogen-into-natural-gas-networks-report2019.pdf.

ii. Jozian, S., \&Vafajoo L. (2018). Mathematical modeling of the gas hydrate formation in a $90^{\circ}$ elbow utilizing cfd technique. Chemical Engineering Transactions, 70, 2167-2172 Doi:10.3303/CET1870362

iii. Jujuly, M. M., Rahman, M. A., Maynard, A., \& Addy, M. (2017). Hydrate Induced Vibration in an Offshore Pipeline. SPE Annual Technical Conference and Exhibition.Doi:10.2118/187378-ms.

iv. Kaczynski, S., Łaciak, M., Olijnyk, A., Szurlej, A., \&Włodek, T. (2019). Thermodynamic and Technical Issues of Hydrogen and Methane-Hydrogen Mixtures Pipeline Transmission. Energies, $12(3)$, 569. doi:10.3390/en12030569

v. Meng, B., Gu, C., Zhang, L., Zhou, C., Li, X., Zhao, Y., ... Han, Y. (2017). Hydrogen effects on X80 pipeline steel in high-pressure natural gas/hydrogen mixtures. International Journal of Hydrogen Energy, 42(11), 7404-7412. doi:10.1016/j.ijhydene.2016.05.145.

vi. Melaina, M. W., Antonia, O., \&Penev, M. (2013). Blending hydrogen into natural gas pipeline networks: A review of key issues. National Renewable Energy Laboratory Technical Report. Retrieved from http://www.osti.gov/bridge.

vii. Neto, T. E., Rahman, M. A., Imtiaz, S., \& Ahmed, S. (2016). Numerical flow analysis of hydrate formation in offshore pipelines using computational fluid dynamics (CFD). Polar and Arctic Sciences and Technology; Petroleum Technology, 8. doi:10.1115/omae2016-54534

viii. Sloan, E. D, Koh, C. A, \& Sum, A. K. (2010). Natural gas hydrates in flow assurance. New York: Elsevier.

ix. Stewart, H.B., \&Wendroff, B. (1984). Two-phase flow: models and methods. J. Comput. Phys. 56(3):363-409, 1984.

x. Thorley, A. R. D., \&Tiley, C. H. (1987). Unsteady and transient flow of compressible fluids in pipelines-a review of theoretical and some experimental studies. International Journal of Heat and Fluid Flow, 8(1), 3-15. Doi:10.1016/0142-727x(87)90044-0.

xi. Wang, Z., Zhang, J., Chen, L., Zhao, Y., Fu, W., Yu, J., \& Sun, B. (2018). Modeling of hydrate layer growth in horizontal gas-dominated pipelines with free water. Journal of Natural Gas Science and Engineering, 50, 364373. doi:10.1016/j.jngse.2017.11.023

xii. Wlodek, T., Laciak, M., Kurowska, K., \& Wegryzn, L. (2016). Thermodynamic analysis of hydrogen pipeline transportation: Selected aspects. AGH Drilling, Oil, Gas, 33(2). 379-396. Doi: 10.7494/drill.2016.33.2.379

xiii. Zhang, P., Wu, Q., \& Mu, C. (2017). Influence of temperature on methane hydrate formation. Scientific Reports, 7(1). doi:10.1038/s41598-017-08430-y

xiv. Sultan, R. A., Rahman, M. A., Zendehboudi, S., \&Kelessidis, V. C. (2017). CFD Simulation of Three Phase Gas-LiquidSolid Flow in Horizontal Pipes. Proceedings of the ASME 2017 Fluids Engineering Division Summer Meeting FEDSM2017. Doi:10.1115/fedsm2017-69051 